\title{
Pathological plantar response: disturbances of the normal integration of flexor and extensor reflex components
}

\author{
LENNART GRIMBY \\ From the Department of Neurology, Karolinska Institutet, \\ Serafimerlasarettet, Stockholm, Sweden
}

Previous reports (Kugelberg, Eklund, and Grimby, 1960; Grimby, 1963) have presented the results of electromyographic studies of spinal reflexes elicited in healthy subjects by painful brief electric stimuli applied to the plantar surface of the foot. Special attention was paid to the 'reflex pattern' obtained on simultaneous recording in the short hallux flexor and extensor muscles, since the strength of the response in the flexor has been shown to be a sensitive index of the tendency to plantar flexion, and the response in the extensor of the tendency to dorsiflexion of the great toe. The investigations of the reflexes obtained in healthy individuals revealed that the reflex pattern has a basic composition typical of the stimulus site. Thus, stimulation of the hollow of the foot gives rise to activity predominantly in the short hallux flexor, whereas hallux stimulation results in dominant extensor activity; as the stimulus is successively shifted from planta to hallux, the flexor activity is gradually replaced by extensor activity. In an area which was called the 'transition zone' and which roughly corresponds to the ball of the foot, the reflex pattern is composed of alternating flexor and extensor activity of equal strength; the site and width of the transition zone vary from individual to individual. Finally, changes in the subject's attention and expectancy may result in marked, but only occasional, deviations from the basic reflex pattern at a given stimulus site.

The electromyographic responses to stimulation of the planta have previously been studied in certain patients with severe spinal cord lesions (Kugelberg et al., 1960); in these cases the reflex patterns consisted of pure short hallux extensor activity independently of the stimulus site. The present study has been undertaken to investigate the types of deviation from the normal reflex patterns that may appear in subjects with less severe lesions of the reflex mechanism, and a direct analysis of the Babinski sign in the common clinical sense is not within the scope of this work. The material has been chosen so as to give the widest possible range of variations, and no conclusions as to the frequency of the different types of deviation in unselected pathological material can be drawn from the results.

\section{MATERIAL AND METHODS}

The investigation is based on material consisting of two groups of cases with spastic para- or hemipareses, viz. one group of 28 patients with clinically confirmes Babinski responses, referred to as the 'Babinski group' $\vec{A} \vec{A}$ and another group of 12 cases in which the clinicaP $\mathrm{O}$ Babinski response was unexpectedly absent; this group will be referred to as the 'non-Babinski group'. Since the reflex patterns studied should be as widely varying as possible, the patients of the first group were selected on the basis of the wide range of variations found in the types? site, and extent of the nervous lesions and in their clinicas $\vec{\bullet}$ reflex responses. In some of the patients of the second of group the Babinski sign had been present in previous phases of their disorders, but at the clinical examination in connexion with this investigation flexor plantar responses were obtained, although slight traces of hallux dorsiflexion might sometimes be observed.

The technique employed was the same as that used in previous investigations (Kugelberg et al., 1960; Grimby, 1963). Thus, various spots on the plantar surface of the foot were exposed to painful repetitive electric shocks over a period of $20 \mathrm{msec}$., and the reflex responses obtained were recorded simultaneously in the short hallux flexor and extensor. All remarkable results were controlled by repeated experiments. The study has been limited to discharges of latencies short enough to rule out voluntary responses, attention being particularly focused on discharges possible to obtain at such brief latencies, i.e., below $100 \mathrm{msec}$. (cf. Grimby, 1963) that they must be presumed to be mediated by spinal reflex arcs.

When selecting suitable subjects for the investigation, atrophic short toe extensors were found to be common among persons suffering from pronounced central pareses of long duration, and in several cases the electromyogram revealed significant peripheral nerve lesions. The same observation was made by Landau and Clare (1959) in their studies of traumatic paraplegia. No 
attempt was made to find out the cause of the peripheral nerve lesions, which may, however, be secondary to the forced inactivity of these patients. Subjects with atrophic short hallux extensors have not been included in the material since the extensor activity obtained in such cases is no satisfactory index of the tendency to dorsiflexion of the great toe.

\section{RESULTS}

GENERAL PROPERTIES OF THE REFLEX PATTERN On stimulation of the planta in normal individuals, the latency of the first discharges in the short hallux muscles may be as brief as $55 \mathrm{msec}$, the reflex attains its maximum strength almost immediately, and after a few hundred milliseconds only little activity is left, at least after habituation. In the pathological cases the latency values were fundamentally the same but there was often a progressive build-up of the reflex strength and a much longer duration of the activity elicited.

When a stimulus gives rise to reflexes both in the short hallux flexor and extensor, the normal discharges have not the same latency but are alternating. Also in the pathological cases such reciprocal discharges were generally observed in the two antagonists. In two cases, however, simultaneous bursts of activity regularly occurred in the short hallux flexor and extensor; the absence of a distinct reciprocity in these cases may be explained by the investigation by Holmqvist and Lundberg (1961) on decerebrate cats, showing that the reciprocity between flexor and extensor motor neurons may be disturbed at a suprasegmental level.

In normal individuals, cerebral factors may influence the basic composition of the reflex pattern obtained at a given stimulus site and strength. Such variations, due to changes in the subject's attention and expectancy, were observed also in the pathological material, except in those extreme cases where pure extensor activity was induced independently of the stimulus type. In the present paper, however, only the average pattern typical of a given stimulus site and strength in a given individual will be considered.

STIMULATION OF THE MIDDLE OF THE PLANTA In normal individuals short hallux flexor activity is by far the most dominant in the reflex pattern on stimulation of the middle of the planta, and significant extensor activity is regularly obtained only in subjects with a very proximal transition zone.

Babinski group In the Babinski group practically all transitional forms, from the normal pure short hallux flexor to the extreme pathological pure

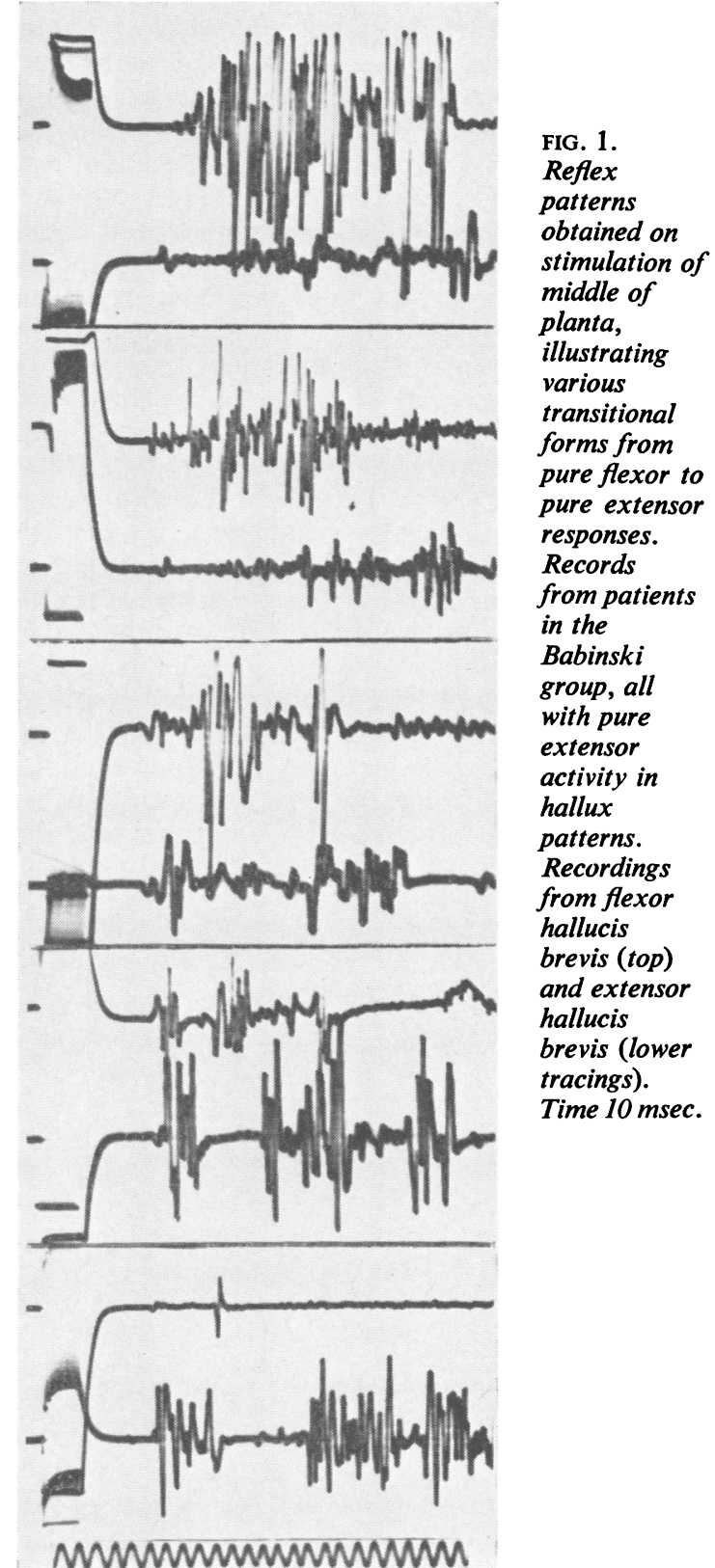

extensor reflex, were observed (Fig. 1). Almost pure flexor responses were obtained in a remarkably large group (nine cases), even including subjects who at the clinical examination exhibited brisk Babinski signs. Pure extensor responses were obtained in a small group of patients (four cases) with severe lesions of the spinal cord but were not a characteristic feature in this type of lesion. In the remaining 15 
cases in the Babinski group the reflex patterns consisted of various combinations of flexor and extensor discharges, as a rule alternating, and closely resembling those obtained on stimulation near the transition zone in normal subjects. The initial and the later parts of the pattern often differed somewhat, and in some cases two different components could be distinguished, viz., either an initial part with dominant flexor activity and shorter latency than $100 \mathrm{msec}$., and a distinctly separate later part with dominant extensor activity and about twice as long latency (four cases, cf. Fig. 3A), or an initial part with dominant extensor and a later part with dominant flexor activity (two cases, cf. Fig. 3B).

Non-Babinski group In the non-Babinski group the extensor activity in the planta pattern was often stronger than in a typical normal case. In eight of the cases it was, however, not stronger than in normal individuals with a proximal transition zone, but in the remaining four cases the plantar pattern differed significantly from normal and consisted of an initial part of dominant extensor and a later part of dominant flexor activity. As was to be expected, no plantar patterns with long-lasting dominant extensor activity, viz., patterns resulting in protracted dorsiflexion of the great toe, were observed in this group.

No opportunity was provided to observe the transformation of normal plantar patterns into pathological patterns, but four patients could be followed up while their plantar responses changed from extensor to flexor; in the course of their recovery the receptive field of the extensor reflexes was successively reduced in the direction of the hallux ball and the extensor activity in the plantar pattern gradually subsided and in two cases finally disappeared. In three of these cases the development of the plantar pattern during recovery can best be illustrated by Fig. 1 as read inversely, i.e., from bottom to top. In the fourth case (Fig. 3A), the pathological plantar pattern consisted of an initial component with dominant flexor activity and a later part with dominant extensor activity; in the course of the recovery of the patient this later part disappeared completely while the initial part remained unchanged.

STIMULATION OF LATERAL SIDE OF THE PLANTA In normal individuals the same reflex pattern is generalls $\omega$ obtained on stimulation of the lateral as of th $\vec{A}$

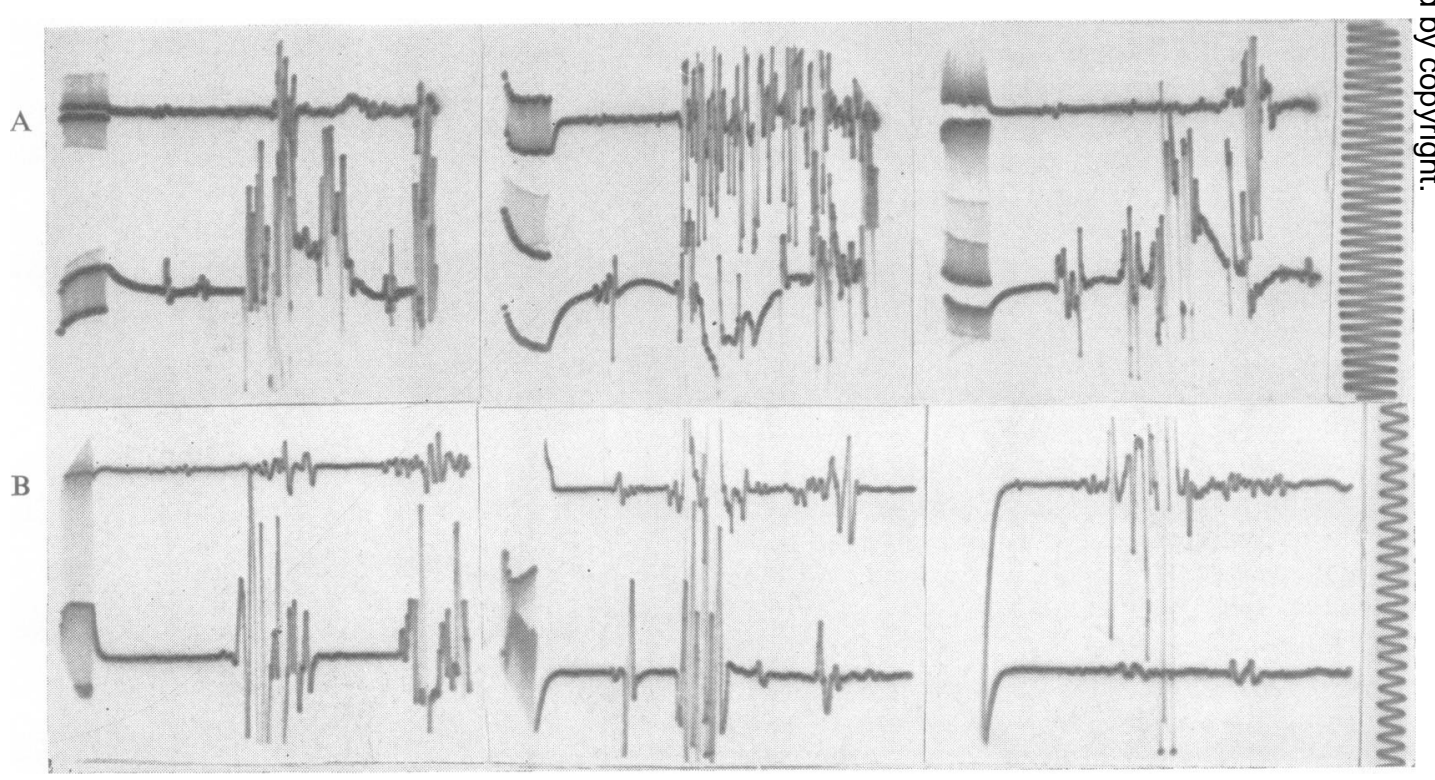

FIG. 2. Changes in reflex patterns on shift of stimulus from middle to lateral side of planta. Stimuli applied to hallux ball (left-hand column), middle of planta (middle column), and lateral side of planta (right-hand column). Recordings from flexor hallucis brevis (top) and extensor hallucis brevis (lower tracings). Time 10 msec.

Case A On lateral planta stimulation, pattern predominantly extensor, thus resembling hallux pattern; on medial planta stimulation, pattern predominantly flexor.

Case B On lateral planta stimulation, pattern predominantly flexor; on medial planta stimulation, pattern predominantly extensor, thus resembling hallux pattern. 
middle or medial side of the planta. If the stimulus is applied to the anterior parts of the sole there is, however, often a greater tendency to extensor activity on medial than on lateral stimulation. In the pathological material, stimulation of the lateral side resulted in the same range of variations as were observed on stimulation of the middle of the planta, viz., from pure flexor to pure extensor responses. In most cases no definite changes were induced by shifting the stimulus from the medial to the lateral side of the planta. In the Babinski group, however, five cases exhibited a marked increase of extensor activity (Fig. 2A) which agrees with the well-known clinical observation that the Babinski sign is more readily evoked from the lateral than from the medial side of the planta. Two cases were anyhow observed where extensor activity was dominant on medial and flexor activity on lateral stimulation (Fig. 2B); although contrary to clinical experience, these findings are less surprising in view of the fact that extensor activity is normally more readily elicited from the medial than from the lateral side of the planta.

STimulation OF THE HALluX Ball Normally, short hallux extensor activity is strongly dominant in the hallux pattern; pronounced flexor activity occurs only in individuals with a very distal transition zone. Also in most pathological cases extensor activity was by far dominant in the reflex patterns induced by hallux stimulation, but signs of flexor activity often appeared which, although not stronger than is normal in subjects with distal transition zones, were anyhow remarkable insofar as they occurred also in subjects with a considerably reduced flexor activity in the plantar pattern. In some cases the flexor activity was slightly more prominent in the early part of the hallux pattern and in other cases in its later part. In three cases, all in the Babinski group, weak hallux stimulation regularly resulted in patterns throughout dominated by flexor activity and even in a distinct plantar flexion of the great toe, a reaction never observed in any of the normal subjects examined.

CONTRAST BETWEEN PLANTA AND HALLUX PATTERNS In normal individuals, flexor activity is strongly dominant in the planta pattern and extensor activity strongly dominant in the hallux pattern, and the contrast between the two patterns is almost maximal, except in subjects with extremely broad transition zones. In most pathological cases, both in the Babinski and the non-Babinski group, this contrast was reduced or even abolished.

In cases where the reflex pattern consisted of two distinctly separate components, the contrast between
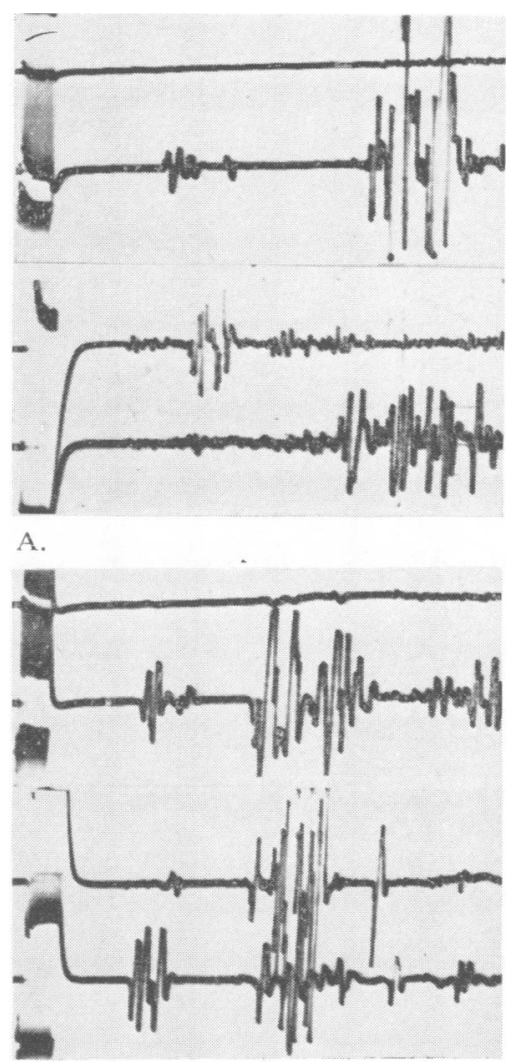

B.

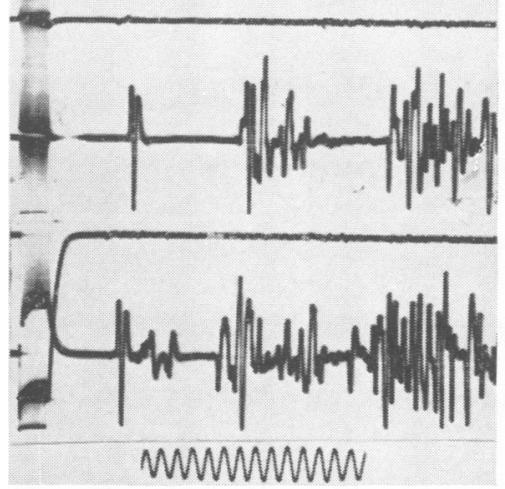

C.

the planta and hallux patterns might be normal in the initial part of the pattern but abolished in its later part (Fig. 3A); conversely, the first part might consist of stereotyped extensor activity whereas the later component had a normal differentiation (Fig. 3B). In some cases, finally, a shift of the stimulus site did not result in any change, either in
FIG. 3 .

Initial and later components of reflex patterns exhibiting significant differences. Upper records in $A, B$, and $C$, hallux stimulation; lower records stimulation applied at middle of planta.

Recordings from flexor hallucis brevis (top) and extensor hallucis brevis (lower tracings). Time $10 \mathrm{msec}$.

Case A

Differentiation of initial reflex component typical of stimulus site, later component stereotyped extensor activity.

Case B Initial reflex component stereotyped extensor activity, later component normal differentiation.

Case C Both reflex components pure extensor, independently of stimulus site. 


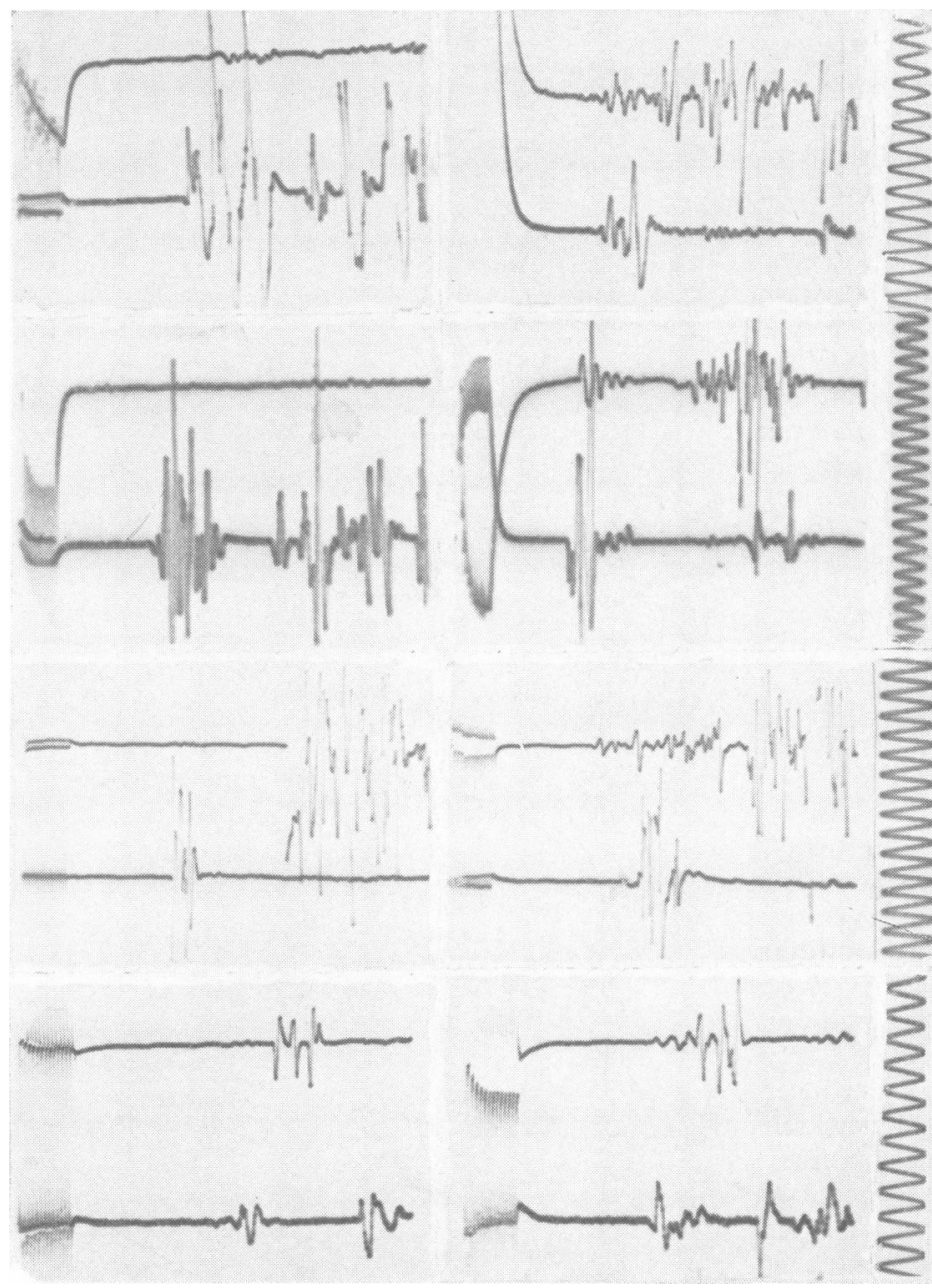

FIG. 4. Varying amounts of contrast between hallux and planta patterns despite similar planta patterns.

Stimuli applied to hallux ball (left-hand column) and middle of planta (right-hand column).

Recordings from flexor hallucis brevis (top) and extensor hallucis brevis (lower tracings). Time $10 \mathrm{msec}$.

the early or in the later part of the reflex pattern (Fig. 3C).

The contrast between planta and hallux patterns may vary considerably, i.e., be more or less pronounced, in cases with rather similar planta patterns (Fig. 4). Besides, there may be a marked contrast between the two patterns although extensor activity is strongly dominant in both; or the two patterns may be similar although there is no general extensor predominance. There is thus no direct relationship between the amount of contrast between the two reflex patterns and the increased level of extensor activity in the planta pattern.

SIGNIFICANCE OF STIMULUS STRENGTH With increasing stimulus intensity the strength of the short hallux extensor reflex normally increases at a somewhat faster rate than the flexor reflex. On an average, this differentiation was more pronounced in the pathological material, especially in the Babinski group, and in one case in this group an increase of the stimulus strength caused not only a relative but an absolute weakening of the flexor reflex (Fig. 5A). In this case the reflex patterns obtained were the same independently of the stimulus site, but flexor activity was dominant on weak. and extensor activity on strong, stimulation; while the normal $\frac{T}{0}$ pattern is almost completely determined by the stimulus site, the stimulus strength was altogether $N$ decisive in this case.

Two cases in the Babinski group formed an exception to the general tendency to increased 


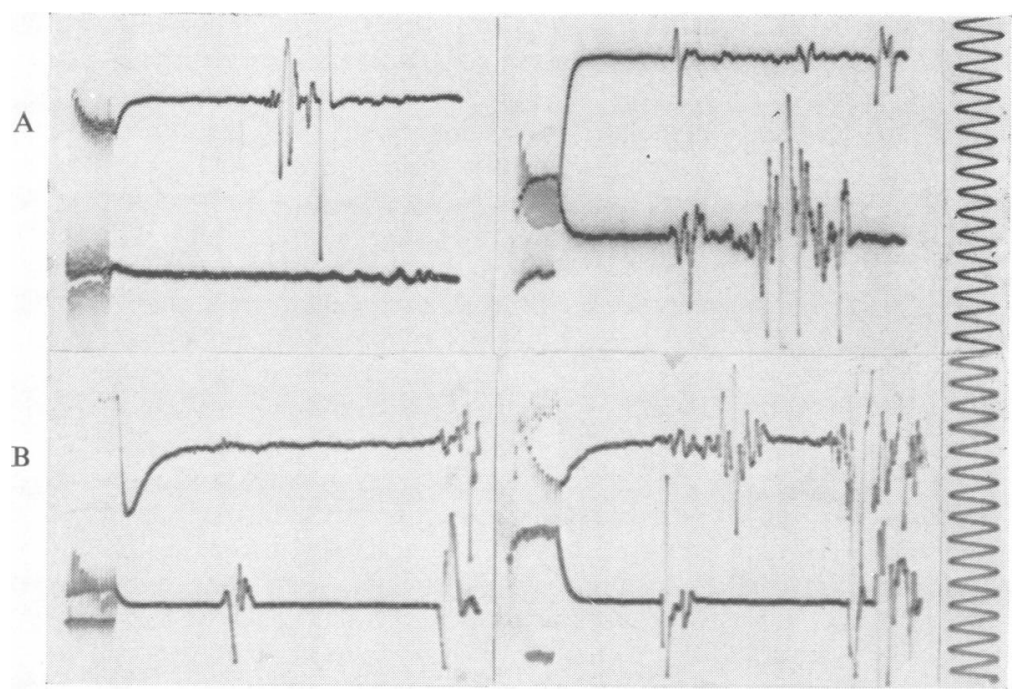

FIG. 5. Influence of stimulus strength on reflex pattern. Left-hand column weak, righthand column strong stimulation. Recordings from flexor hallucis brevis (top) and extensor hallucis brevis (lower tracings). Time 10 msec.

Case A Pattern predominantly flexor on weak, extensor on strong, stimulation (records from anterior part of planta but results independent of stimulus site).

Case B Pattern predominantly extensor on weak, flexor on strong, stimulation (stimulus applied to lateral side of planta). extensor predominance with increasing stimulus strength; in these cases very weak planta stimulation resulted in pure extensor activity, although stronger stimulation provoked dominant flexor activity (Fig. 5B); in one of the cases, however, extensor activity was again dominant on application of very strong stimuli.

\section{DISCUSSION}

This investigation of the integration of the short hallux flexor and extensor reflexes in pathological cases is a direct sequel to the previous corresponding study performed on healthy individuals (Grimby, 1963), and the results should thus be correlated to those obtained in normal cases.

Even in a normal material, ruling out all cases of clinically atypical reflexes where an injured reflex mechanism may be suspected, significant deviations from the average normal reflex pattern can be observed, and it was presumed that an unselected population of apparently healthy individuals would include single cases exhibiting still more pronounced deviations than those actually found in the recent investigation. Since there is, however, no safe method of judging whether the reflex mechanism is actually intact in such cases, no exact limits can be drawn for the normal variations. It would anyhow appear to be justified to assume that very marked deviations from normal observed in a high proportion of the pathological cases are, in fact, caused by the nervous disorders present, and the following discussion will be based on this assumption.

Since the extensor activity in the reflex pattern obtained on stimulation of the planta in subjects with pathological reflexes gradually subsides and sometimes disappears as the patients recover, a direct relationship must be presumed to exist between the varying degrees of extensor activity and the degree of pathological change in the reflex mechanism. The extensor activity sometimes present in the planta pattern in all normal cases is only occasional and disappears on repeated stimulation; the intact reflex mechanism may thus, in contrast to that injured, be able to correct deviations from the normal reflex pattern. As, however, a relatively weak but constant extensor activity may sometimes occur in the plantar pattern also in single normal cases, this type of reflex pattern must be considered to be either a normal variant or the effect of an injured reflex mechanism. No exact margin can thus be drawn between normal and pathological plantar patterns.

When a pathological plantar pattern is successively converted into a normal reflex type, the extent of the receptive field of the extensor reflex gradually shrinks in the direction of the hallux ball, and it must be presumed that, inversely, the receptive field successively spreads to a larger area as a normal pattern is converted into a pathological; this concept would lend further support to the view advanced by Kugelberg et al. (1960) that the extensor reflex obtained on hallux stimulation in normal cases is fundamentally the same as that elicited on planta stimulation in pathological cases. Since in some cases pronounced extensor activity can be elicited solely by lateral, and in other cases solely by medial, planta stimulation, there must be individual 
differences in the extension of the receptive field of the extensor reflex, and these may be accounted for either by constitutional individual differences or by differences in the nature of the nervous lesions involved.

Two distinctly separate components may be distinguished in the reflex pattern; the two responses may differ in habituation tendency (cf. Grimby, 1963) and in composition (cf. Fig. 3) and are probably transmitted in somewhat different ways. The latency of the first response in the reflex pattern is so short that it must be of spinal origin and mediated by A-fibres. Kugelberg (1948) has shown that the pathological plantar response may consist of an early A- and a later C-fibre response, but the second response in the reflex pattern discussed in this work has such a short latency that it cannot be mediated by $\mathrm{C}$-fibres. The longer latency of the second response is more likely to be due to a longer central reflex time than to slower afferent impulses, but whether this response is of spinal or of cerebral origin cannot be decided judging by the latency values; nor can it be ruled out that it is of a different origin in different cases. That the deviation from normal observed in pathological cases may be limited either to the first or to the second response indicates that a lesion may affect one response, leaving the other unaffected, and this differentiation is probably due to the difference in transmission, whatever this difference may be.

In cases where a Babinski response is obtained on clinical stimulation, the plantar pattern may often be normal; conversely, markedly deviating patterns may be observed in pathological cases with clinically normal reflexes. It would seem as though the brief stimulus used in the experiments favours the initial purely spinal response, whereas the later phenomena are more apparent in the long-lasting stimulation used in the clinical examination. Besides, the stimulus strength may be of fundamental significance for the composition of the plantar pattern (Fig. 5), and the tendency to dominant extensor activity seems to be least pronounced on stimuli of medium strength and to increase both with stronger (Fig. 5A) and weaker (Fig. 5B) stimulus intensity. This is in good agreement with the finding that in normal cases marked extensor activity in the plantar pattern is more common on very strong or very weak stimulation than when stimuli of medium strength are set in.

When the reflex mechanism is intact, the reflex pattern is closely correlated to the site of the stimulus so that the resulting response always represents the most appropriate defence reaction; when it is injured this ability may be reduced or abolished, and the reflex pattern is then less adaptive to the stimulus site. In some pathological cases the reduction of the contrast between hallux and planta patterns is more conspicuous than the general on increase of the extensor activity in the reflex system; in other cases, however, the latter phenomenon is prevalent. This discrepancy may be too large to be explained by constitutional individual differences $\stackrel{\rho}{5}$ (Grimby, 1963) and must be due to different types

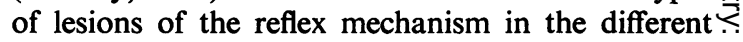
cases. On the basis of the results obtained in the $\vec{F}$ recent study on normal subjects it was assumed that the control of the spinal reflex centre is exerted by one pathway determining the general relation between extensor and flexor activity and another determining

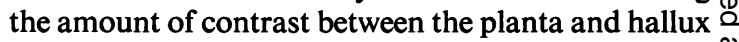
patterns; it seems reasonable to assume that these $\%$ pathways may, independently of one another, be $\vec{\circ}$ more or less affected by the pathological process. That hallux patterns with dominant flexor activity $\vec{\omega}$ may be observed in single pathological cases, even where clinical Babinski signs are present, may then be explicable on the ground that the contrast in between hallux and planta patterns has been abolished without any simultaneous increase of the extensor activity being apparent.

The individual deviations from the normal refle 0 patterns observed in pathological conditions may so manifold and varying that they must be presumed to be influenced not only by the degree of the lesiogico but also by the type and site of the injury presen However, a positive approach to these problen $\vec{\theta}$ requires studies of a substantial number of case $\mathscr{C}_{\bar{S}}$ with well-defined injuries of the nervous system, and in this connexion the method described above may be a useful tool, as permitting a more exact and detailed reflex analysis than that used in the clinical routine. It should be emphasized, however,

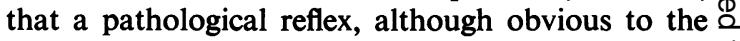
clinician in the course of the ordinary tests, may not appear as such when studied by the new technique; on the other hand, this method may in other cases disclose deviations from normal not apparent at the clinical examination, and may thus be of some practical value as a complement of the clinical tests.

\section{SUMMARY}

The cutaneous reflexes of the foot were investigated 0 in a group of 40 patients with spastic hemi- or parapareses, 28 of whom exhibited clinical Babinski signs. Painful stimuli consisting of a series of repetitive electric shocks delivered over a period of $\mathscr{N}$ $20 \mathrm{msec}$. were applied to various points on the $N$ plantar surface of the foot. The 'reflex patterns' N obtained on simultaneous electromyographic record- 
ing in the short hallux flexor and extensor were compared with those observed in a group of normal cases previously studied.

In the pathological material the reflexes obtained on stimulation of the planta may exhibit a wide range of transitional forms from the typical normal pure short hallux flexor reflex to the extreme pathological pure short hallux extensor reflex; a direct relationship is presumed to exist between the degree of extensor activity in the plantar pattern and the degree of pathological change in the reflex mechanism. However, no exact margin can be drawn between normal and pathological plantar patterns, since a relatively weak but constant extensor activity may sometimes occur in the plantar pattern also in single normal cases.

The pathological reflex pattern may consist of two distinctly separate components. The extensor activity in the plantar pattern, viz., the deviation from normal, is in some cases limited to the early and in other cases to the later component. The early response is of such a short latency that it must be a purely spinal reflex. The origin of the later response cannot be determined judging by its latency but it is assumed to be transmitted in such a way that it may be affected by the pathological process independently of the early response.

In pathological cases, the normal contrast between dominant flexor activity in the plantar pattern and dominant extensor activity in the hallux pattern is reduced, although in some cases more, and in other cases less, than was to be expected from the amount of extensor activity in the plantar pattern; it has been assumed that different pathways may be involved in the suprasegmental reflex control and that these pathways may, independently of one another, be more or less affected by the pathological process.

The individual variations found in the pathological material are too manifold to be ascribed solely to lesions of varying degrees of severity; it has been assumed that different types of lesion of the reflex mechanism may result in different types of deviation from the normal reflex pattern.

\section{REFERENCES}

Grimby, L. (1963). J. Neurol. Neurosurg. Psychiat., 26, 39.

Holmqvist, B., and Lundberg, A. (1961). Acta physiol. scand 54, suppl., 186.

Kugelberg, E. (1948). Brain, 71, 304.

, Eklund, K., and Grimby, L. (1960). Brain, 83, 394.

Landau, W. M., and Clare, M. H. (1959). Ibid., 82, 321. 